\title{
Recursos Tecnológicos: metodologia de ensino para o desenvolvimento de competências e habilidades para comunicação em Língua Inglesa.
}

\author{
Technological Resources: teaching methodology for the development of \\ competences and skills for comunication in English.
}

Poliane Pimentel dos Anjos ${ }^{1 *}$, Prisna Jamile Santos Leder ${ }^{1}$, Bruna Kerolly Epifanio Martins ${ }^{1}$, Daniela Sias ${ }^{1}$, Paula Vitória Matos Menezes ${ }^{1}$, Rafael Silva dos Santos Júnior ${ }^{1}$

\begin{abstract}
RESUMO
Atualmente, os recursos tecnológicos estão cada vez mais presentes, não só no cotidiano social, mas também têm se tornado ferramentas pedagógicas indispensáveis para melhoria do processo de ensino-aprendizagem. Partindo dessa reflexão, foi observado um bom rendimento de alguns alunos que utilizam frequentemente a internet e seus recursos para aprender a língua inglesa, em contraposição aqueles que não utilizam nenhum meio para a própria aprendizagem e apresentam dificuldades na disciplina. Assim, de acordo com as observações feitas foi possível constatar uma melhoria no rendimento dos estudantes com o apoio das ferramentas tecnológicas. Em virtude disso, a ideia de se fazer um estudo para avaliar os impactos da inserção de ferramentas tecnológicas como método auxiliadores da aprendizagem autônoma dos discentes se mostrou muito promissora, tendo em vista a utilização constante de smartphones e tablets por eles.
\end{abstract}

Palavras-chave: Apredizagem de Lingua Inglesa; Autonomia; Recursos Tecnologicos; Habilidades.

\begin{abstract}
Currently, technological resources are increasingly present, not only in social daily life, but also, it has becoming essential pedagogical tools for improving the teaching-learning process. Based on this reflection, a good performance of some students who frequently use the internet and its resources to learn the English language was observed, in contrast to those who do not use any means for their own learning and present difficulties in the subject. Thus, according to the observations made, it was possible to see an improvement in student performance with the support of technological tools. As a result, the idea of carrying out a study to assess the impacts of the insertion of technological tools as an aiding method for the autonomous learning of students proved to be very promising, in view of their constant use of smartphones and tablets.
\end{abstract}

Keywords: English Knowledge; Autonomy; Tecnologycal Resources; Habilities.

\footnotetext{
${ }^{1}$ Secretaria de Estado de Educação do Amazonas - SEDUC/AM.

*E-mail: poliane.pimentel@gmail.com
} 


\section{INTRODUÇÃO}

O projeto proposto traz uma reflexão a partir das questões que envolvem a apredizagem na aquisição de um novo idioma e como ele se faz presente no cotidiano dos estudantes, preparando-os para compreender assuntos partindo do geral, para o particular, onde relacionarão os conteúdos contextualizados nas atividades práticas, com os temas escolares e sua vivência local. Com base nisso, uma das relevâncias de se dirigir este estudo foi o conhecimento sobre a existência de diversas discussões entre os estudiosos acerca do potencial de novos Aplicativos (Apps) para os trabalhos didáticos-pedagógicos contemporâneos, considerarando que elas permitem que sejam criadas situações de aprendizagens ricas, complexas e diversificadas (PERRENOUD, 2000; CENSI, 2017; FIGUEIREDO \& NANTES, 2017; ESTEVES \& RIBEIRO, 2019). Desta maneira, há um amplo crescimento em pesquisas direcionadas para o uso de recursos tecnologicos em sala de aula de língua estrangeira.

Pinto (2004) esclarece que há a exigência de um domínio, cada vez maior, de conhecimentos e de habilidades para lidar com tais recursos, pois eles impõem novas concepções de educação, escola e ensino. Apoiando essa proposição, Prieto (2011) ressalta a importância do uso de tecnologias em ambientes educativos, de modo a adaptar a realidade do sistema educacional, aproveitando as potencialidades dos recursos tecnológicos no processo de aprendizagem. Assim, a proposta desse projeto alia atividades práticas para relacionar os conteúdos curriculares da língua inglesa (LI) do $7^{\circ}$ ano do ensino fundamental com linguagem do dia-a-dia, estimulando a reflexão e colaborando para a melhoria do ensino.

Apesar de alguns trabalhos terem sido desenvolvidos nessa área, há escassez de estudos que abordem os conteúdosrelacionados especificamente a uma série de ensino na educação básica, e que priorizem atividades teóricas e práticas alternativos para contextualização da linguagem falada no cotidiano do aluno, em se tratando da LI e das quatro habilidades. Ademais, até o momento não foi desenvolvido na escola pretendida, nenhum projeto nesse contexto, sendo uma ação inédita que tratou evidência e incentivo a outras iniciativas que beneficiem a melhoria do ensino, 
também atenderá a missão da escola em questão, que é educar para formar pessoas críticas, conscientes e capazes de transformar o meio em que vivem.

Nessa perspectiva, o presente estudo foi desenvolvido no âmbito do Programa Ciência na Escola (PCE), fomentado pela Fundação de Amparo à Pesquisa do Estado do Amazonas (Fapeam) com a finalidade de despertar no aluno o desejo de aprender dinamicamente a língua inglesa utilizando as mais diversas tecnologias, para que ele possa sentir-se familiarizado na aprendizagem desse idioma e saber comunicar-se sem proscrever as reais necessidades de se estar em constante evolução no conhecimento da língua.

\section{METODOLOGIA}

No presente estudo, além de diagnosticar quais são (e se são) as ferramentas (aplicativos, software, etc.) utilizadas pelos estudantes, buscou-se também, empregar e avaliar o potencial da utilização dos Apps Duoling, Reverso e Wlingua, como ferramentas didático-pedagógica para o avanço do ensino-aprendizagem da língua inglesa. É de suma importância destacar que em estudos voltados ao ensinoaprendizagem de línguas estrangeiras, no que diz respeito às quatro habilidades linguisticas, ler, falar, ouvir e escrever ( read / speak / listen / write), mediadas por recursos tecnológicos são necessários para as melhorias das práticas e para a ampliação desse campo específico.

Desta forma, a presente pesquisa foi desenvolvida com os alunos do ensino fundamental dois da Escola Estadual Balbina Mestrinho/Urucará - AM, visando, por meio desse projeto, facilitar a aprendizagem dos estudantes em relação aos conteúdos escolares de LI, para viabilizar a aplicação do conhecimento adquirido em seu cotidiano, e assim, compreender a importância de conhecer um novo idioma.

O estudo foi desenvolvido durante seis meses e contou coma participação de quatro alunos bolsistas do projeto, os quais envolveram-se primeiramente em aulas de LI, em seguida tiveram contato com diversos Apps que trabalham com a aprendizagem desse idioma. Após isso, os aplicativos Duolingo, Reverso Context e Wlingua foram os três aplicativos selecionados para serem testados e analisados posteriormente pelos demais 
estudantes. Participaram, voluntariamente desse estudo 120 alunos do $6^{\circ}$ ao $9^{\circ}$ ano do Ensino Fundamental, da E.E. Balbina Mestrinho.

Os resultados do levantamento teórico e das considerações que levaram os bolsistas do projeto a escolha desses três aplicativos, foram por eles apresentados aos demais estudantes, no auditório da referida escola, com auxílio de slide e banners. Posterior a isso, aplicou-se o instrumento de pesquisa que foi um questionário composto por dez perguntas relacionadas a utilização dos aplicativos, sendo seis de cunho quantitativo e quatro de cunho qualitativo.

A etapa de finalização, do projeto, deu-se mediante a realização de uma culminância, aberta ao público, junto a mais dois projetos também aprovados e desenvolvidos no âmbitos do PCE 2021 no município de Urucará.

\section{RESULTADOS E DISCUSSÕES}

Durante a aplicação do projeto no auditório da escola, foram feitas aos estudantes, explanações acerca dos aplicativos analisados e, em seguida, os participantes responderam algumas perguntas relacionadas ao funcionamento de cada aplicativo. Nessa perspectiva, na Figura 1 são mostradas as respostas dadas pelos estudantes quando questionados sobre quais dos aplicativos analisados neste estudo já eram de seu conhecimento ou uso cotidiano.

Figura 1: Disseminação de uso dos aplicativos.

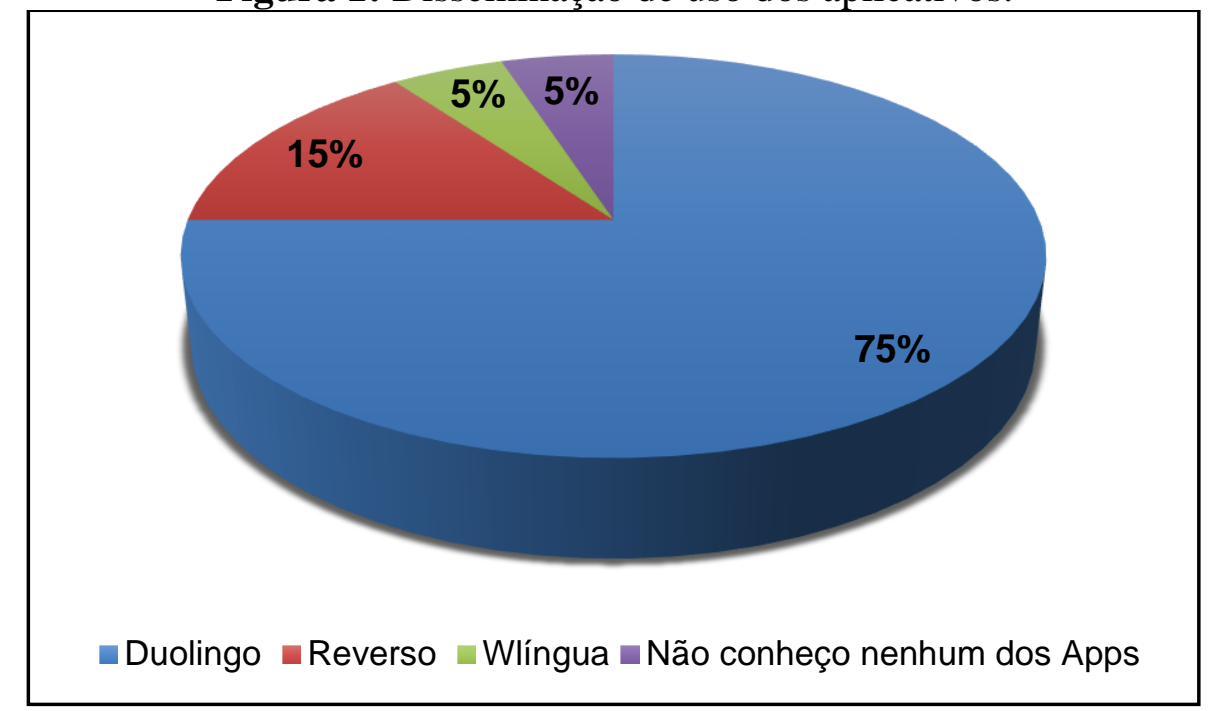

Fonte: Dos Anjos et al. (2021). 
De acordo com o mostrado na Figura 1, $75 \%$ dos estudantes já apresentavam conhecimento prévio sobre o App Duolingo, ainda que não tivessem feito o uso efetivo do mesmo. O Duolingo é um dos Apps mais popular e baixados no mundo por pessoas que procurando aprender outro idioma e, em virtude disso, ele vem ganhando destaque na área acadêmica. Posto isso, estudiosos constatam a influência do aplicativo e ressaltam os pontos positivos do emprego do Duolingo como ferramenta didática para auxiliar o aprendizado dentro ou fora da sala de aula (DUARTE, ALDA \& LEFFA, 2016; SOARES, 2018; SOARES \& LIMA, 2019).

A interface gráfica (Layout) apresenta os elementos gráficos de cada aplicativo, sendo ela fundamental para a compreensão da funcionalidade de suas ferramentas pelo público-alvo, a qual depende da disposição gráfica dos elementos visuais contidos nelas (HARRES, RUBIN \& BRAWERMAN-ALBINI, 2020). Deste modo, na Figura 2, são mostrados os resultados comparativos referentes interfaces dos aplicativos Duolingo, Reverso e Wlingua, segundo os estudantes.

Figura 2: Compreensão do Layout dos Aplicativos.

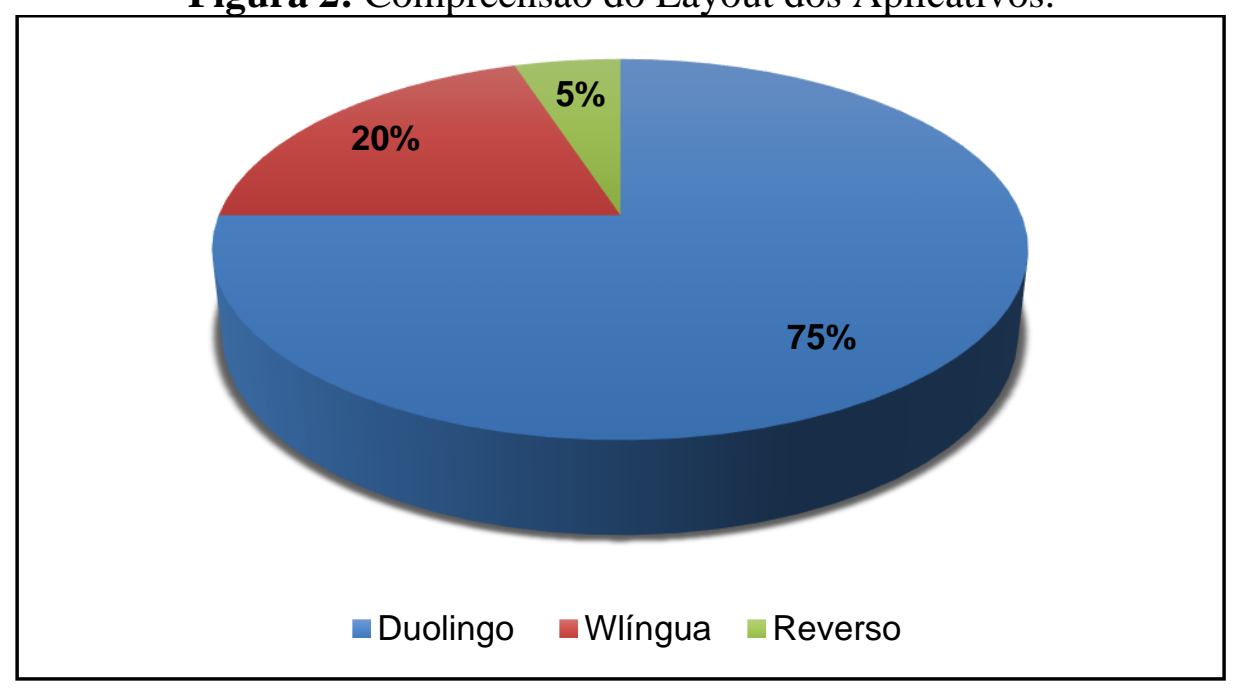

Fonte: Dos Anjos et al. (2021).

Após manusear e comparar os três Apps analisados neste estudo, apenas $5 \%$ dos participantes consideraram o Layout do App Reverso claro e de simples compreensão. Outros $20 \%$ dos alunos julgaram que a interface do App Wlingua foi a que apresentou maior clareza e simplicidade de domínio. Os aplicativos educacionais são desenvolvidos com a finalidade de dinamizar o aprendizado do usufrutuário, realizando a análise das informações fornecidas pelo próprio usuário ao realizar suas atividades, as quais podem ser direcionados à modalidade de leitura, vocabulário e atividades de fixação. O Wlingua 
oferece ao seu usuário a possibilidade de compreender o alfabeto e palavras com sotaque americano ou britânico, além de empregar recursos de escrita, leitura, pronúncia, dentre outras (GONÇALVES \& SILVA, 2014).

Frente aos Apps Reverso e Wlingua, o Duolingo foi o mais bem avaliado pelos estudantes, visto que $75 \%$ deles qualificaram seu Layout como o mais didático, dinâmico e eficiente para o ensino-aprendizagem de língua inglesa. A interface gráfica do Duolingo, é constituída por uma ramificação que reúne elementos atrativos para a aprendizagem, com botões e atividades que têm uma apresentação semelhante a um cartum, fazendo com que estudante se desprenda da possível visão punitiva sobre as aulas de tradução (HARRES, RUBIN \& BRAWERMAN-ALBINI, 2020).

Mesmo diante da vasta variedade de aplicativos educacionais direcionados ao ensino-aprendizagem de um novo idioma, o App Duolingo vem ganhando grande destaque por ser um aplicativo dinâmico, de fácil acesso e compreensão, além de consistir em uma plataforma gratuita que viabiliza a criação de grupos de estudos, que estimulam a competição de conhecimento entre os usuários do App, culminando assim, no maior interesse em se aprofundar no estudo de um idioma (SILVA et al., 2017).

Para que o objetivo do desenvolvimento de um aplicativo seja cumprido, é necessário que o mesmo seja de fácil acesso. Desta forma, Silva, Ferreira e Sacramento (2018), destacam que a acessibilidade é o direito que qualquer pessoa tem de usufruir dos produtos e serviços ou informações que pertencem ao cotidiano social. Na Figura 3, é mostrado o resultado referente a acessibilidades das três ferramentas didática analisadas nesta pesquisa.

Figura 3: Acessibilidade dos Aplicativos.

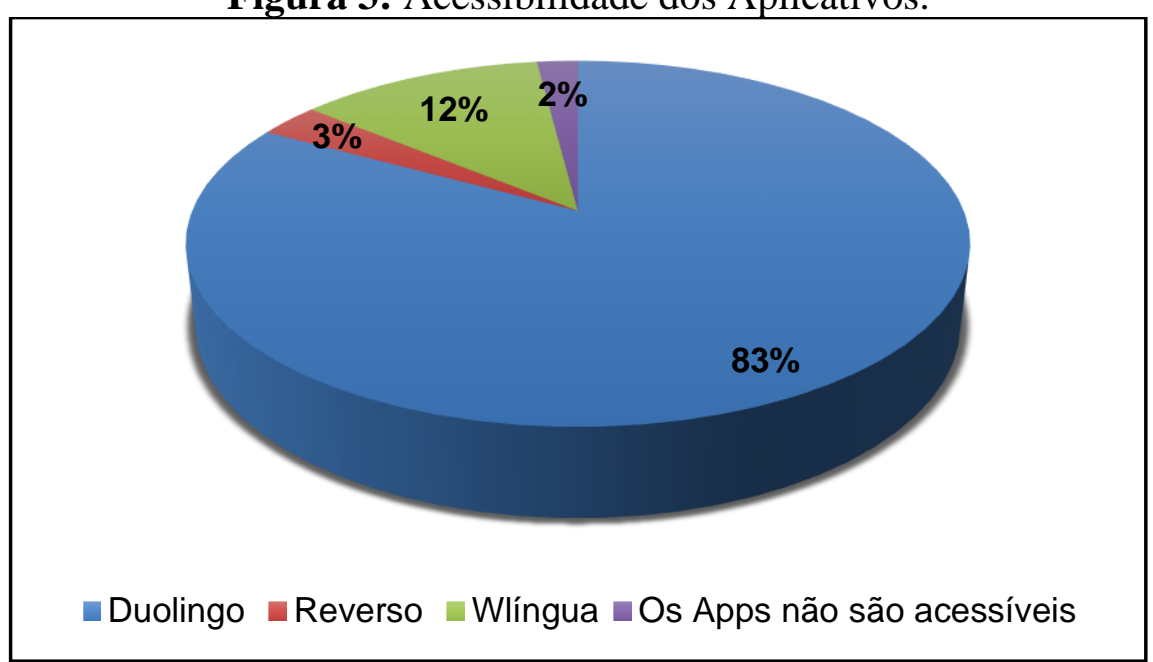

Fonte: Dos Anjos et al. (2021). 
No que concerne à acessibilidade dos Apps, o Duolingo foi considerado por 83 $\%$ dos estudantes como sendo o mais acessível, seguido do Wlingua com $12 \%$, corroborando assim, com o resultado referente a análise da interface gráfica desses Apps, mostrado na Figura 2, que demonstra que o Layout do Duolingo e do Wlingua foram considerados os mais dinâmicos e didáticos, segundo os discentes. O destaque desses Apps deve-se a ampla divulgação que eles vêm ganhando no mundo científico nos últimos anos, visto que diversos autores vêm desenvolvendo estudos voltados para a utilização desses Apps como ferramentas didáticas potencializadoras do ensino-aprendizagem de língua inglesa dentro e fora da sala de aula (GONÇALVES \& SILVA, 2014; COELHO et al. 2016; DUARTE, ALDA \& LEFFA, 2016; FIGUEIREDO \& NANTES, 2017; ALMEIDA, 2020).

O App Reverso foi considerado o de menor acessibilidade, tendo a preferência de apenas $3 \%$ dos discentes envolvidos na pesquisa, e tal fato pode estar diretamente relacionado a difícil compreensão de sua interface gráfica (Figura 2). Para desenvolvimento de um App, vários fatores devem ser levados em consideração e um deles é a forma que o usuário irá interagir com esse App, logo, se forem de difícil acesso, e não possuírem recursos da tecnologia assistiva, não vão ajudar os seus usufrutuários e estes irão encontrar problemas em relação a compatibilidade, desempenho, usabilidade e eficácia na aprendizagem, quando se trata de um App educacional (CARVALHO \& FREIRE, 2017; NETO et al. 2021).

Nessa perspectiva, na figura 4 é ilustrado o reflexo do emprego desses aplicativos no processo de ensino-aprendizagem dos discentes. 
Figura 4: Melhoria na aprendizagem de língua inglesa com o auxílio dos Aplicativos.

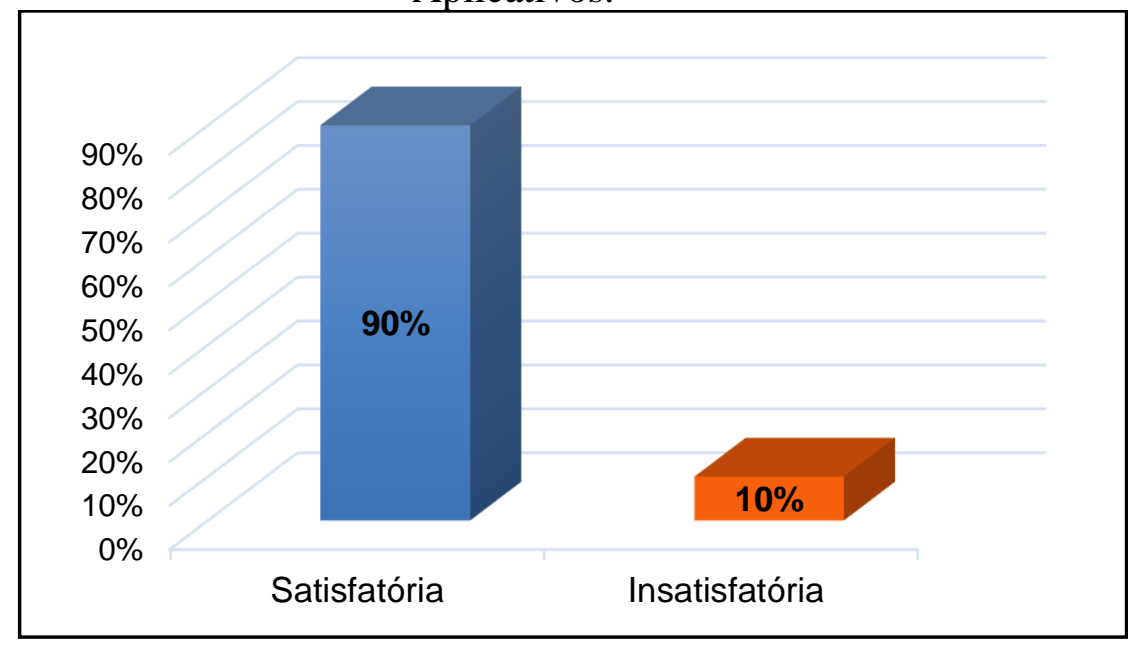

Fonte: Dos Anjos et al. (2021).

Os dados apontam que, em relação a melhoria do aprendizado da língua inglesa com o auxílio dos Apps, que apenas $10 \%$ dos estudantes mencionaram não que tiveram melhoria em seu aprendizado principalmente devido à dificuldade de manuseio dos Apps. Em contrapartida, $90 \%$ dos participantes, que usaram as três ferramentas analisadas, relataram que houve melhoria significativa em sua aprendizagem e consequentemente em seu desempenho no componente curricular melhorou de LI, além disso, elevou o interesse em aprender o referido idioma de forma fluente.

Santana (2016), realizou um estudo empregando o App Duolingo como ferramenta didática para auxiliar o processo de ensino-aprendizagem de língua estrangeira e, segundo os resultados obtidos pelo respectivo autor, o Duolingo é uma ferramenta pedagógica capaz de promover uma aprendizagem significativa para os aprendizes iniciantes de língua estrangeira, visto que este atua como um complemento de ensino.

O desenvolvimento e utilização da tecnologia educacional está em expansão, sobretudo no ensino-aprendizagem de língua inglesa. vive-se hoje, o advento da inserção dos Apps à prática pedagógica de diversos professores, os quais podem promover a facilidade de acesso ao conhecimento de variados idiomas com uma metodologia autônoma de aprendizagem e gerenciável pelo discente que os utilizam de acordo com suas necessidades de estudos. 


\section{CONSIDERAÇÕES FINAIS}

Por meio desse estudo foi possível observar a importância do uso de recursos tecnológicos como ferramentas auxiliadoras no processo de ensino-aprendizagem de língua inglesa no âmbito escolar para a melhoria do desempenho dos estudantes em sala de aula, para o desenvolvimento das quatros habilidades do idioma, bem como para o enriquecimento do vocabulário.

\section{REFERÊNCIAS}

ARAGÃO, R. C. Formação, Reflexão e Tecnologias no Ensino na Bahia. ARAÚJO, J.C.; DIEB, M. (Org.). Letramentos na Web: gêneros, interação e ensino. 1 ed., Fortaleza: Edições UFC, 2009, p.58-82.

ARAUJO, J.; LEFFA, V. Redes sociais e ensino de línguas: o que temos de aprender? São Paulo: Parábola editorial, 2016.

BENSON, P.; VOLLER, P. Autonomy and Independence in Language Learning. London: Longman. 1997. 284p.

CARVALHO L. P.; FREIRE A.P. Native or Web-Hybrid Apps?: An Analysis of the Adequacy for Accessibility of Android Interface Components Used with Screen Readers . Association for Computing Machinery New York NY United States; 2017. Disponível em: <https://dl.acm.org/doi/10.1145/3160504.3160511> . Acesso em: 03/01/2022.

CENSI, L. J. L. Celulares nas escolas: Implicações para as práticas docentes. Revista Linguagem, Ensino e Educação. Criciúma, v. 1, n. 1, p. 1-9, 2017.

COELHO, R. da C.; MOTA, B. C. C.; SILVA, V. M. da C.; NETO, J. M. M. Duolingo: inovação do inglês na escola. Revista do Plano Nacional de Formação de Professores da Educação Básica. Universidade Federal do Piauí, Form@re, v. 4, n. 1, p.142-147, 2016.

DEL MONICO. Michelli de Godoy, As Tecnologias digitais de informação e comunicaçãona aprendizagem autônoma da lingua inglesa. / Michelli de Godoy Del Monico - $201788 \mathrm{f}$.

DIAS, R. Wequests, tecnologias, multiletramentos e formação do professor de inglês para a era do ciberespaço. RBLA, Belo Horizonte, v. 12, n. 4, p. 861-881, 2012.

DICKINSON, L. Learner Autonomy: what, why and how. In. LEFFA, V. J. (Ed.) Autonomy in Language Learning. Porto Alegre: Editora da Universidade/UFRGS, Porto Alegre, 1994, p. 2-12. 
DUARTE, G. B.; ALDA, L.; LEFFA, V. Gameficação e o feedback corretivo: considerações sobre a aprendizagem de línguas estrangeiras pelo Duolingo. Raído, Dourados, MS, v. 10, n. 23, jul./dez, 2016.

ESTEVES, J. R.; RIBEIRO, L. O. M. Aprendizagem de língua inglesa por aplicativos. Revista de Educação à Distância - EmRede, v. 6, n. 1, 2019.

FIGUEIREDO, A. G. F.; NANTES, E. A. S. Ensino e tecnologia: Uma proposta de exploração do Duolingo, via plano de trabalho docente, para o ensino de línguas. Conhecimento Online. Londrina, v. 2, p. 77-101, 2017.

GONÇALVES, J. A.; SILVA, V. Inglês na palma da mão: possibilidades de aprendizagem através dos dispositivos móveis conectados à internet. Revista de Estudos Acadêmicos de Letras. v. 7, n. 1, 2014.

HARRES, V. M.; RUBIN, L.; BRAWERMAN-ALBINI, A. Análise e uso de aplicativos celulares para o ensino de língua inglesa. Revista CBTecle. v. 1, n. 1, 2020.

LEFFA, V. J. Quando menos é mais: a autonomia na aprendizagem de línguas. In: NICOLAIDES, C.; MOZZILLO, I; PACHALSKI, L.; MACHADO, M.; FERNANDES, V. (Orgs.). O desenvolvimento da autonomia no ambiente de aprendizagem de línguas estrangeiras. Pelotas: UFPEL, 2003, p. 33-49

LEFFA, V. J. O ensino de línguas estrangeiras nas comunidades virtuais. In: IV SEMINÁRIO DE LÍNGUAS ESTRANGEIRAS. Anais do IV Seminário de Línguas Estrangeiras. Goiânia: UFG, v. 1, 2002. p. 95-108.

LEFFA, V. J. A aprendizagem de línguas mediada por computador. In: Leffa, V.J. (Org). Pesquisa em linguística aplicada: temas e métodos. 1ed. Pelotas: Educat, v.1, p.11-36.

LITTLE, D. Learner autonomy and human interdependence: some theoretical and practical consequences of a social-interactive view of cognition, learning and language. In B. SINCLAIR, I.; MCGRATH and T. LAMB (Eds). Learner Autonomy, Teacher Autonomy: Future Directions. Harlow: Longman/Pearson Education, 2000, p.15-23.

LITTLE, D. The essence of language learner autonomy: learning a language from inside out. Nordic Conference on Autonomous Learning,11, São Sebastian, 2012.

LITWIN, E. Educação a Distância: temas para Debate de uma Nova Agenda Educativa. Porto Alegre, Artmed, 2001. 109p.

NETO, N. G. S.; MEDEIROS, F. P. A.; ARAÚJO, R. P.; SILVA, A. M. Acessibilidade em dispositivos móveis: uma análise sob a perspectiva das pesquisas em interação humano computador no Brasil. Brazilian Journal of Development, Curitiba, v.7, n.4, p. 3413734150, 2021

PALLOFF, R.; PRATT, K. Construindo comunidades de aprendizagem no ciberespaço: estratégias eficientes para salas de aula on-line. Trad. Vinicius Figueira. Porto Alegre: Artmed, 2002. 
PERRENOUD, P. Dez novas competências para ensinar. Porto Alegre: Artmed, 2000.

PINTO, A. M. As novas tecnologias e a educação. Anais do V Seminário de Pesquisa em Educação, v. 2, n.3. Região Sul, 2004.

PRIETO, M. S. F. A integração da Tecnologia de Informação e Comunicação (TIC) em sala de aula. Anais do Colóquio Interdisciplinar da Licenciatura em Ciências Exatas, 2011.

SILVA, A. B. S.; SILVA, E. B.; COSTA, K. M. S. A; OLIVEIRA, L. C. F. O uso do aplicativo Duolingo em uma turma de Comércio Exterior na Faculdade de Tecnologia da Zona Leste de São Paulo. CBTecLE, v. 1, n. 2, 2017.

SILVA, A. C. O Facebook como rede social de aprendizagem e ensino de língua inglesa. Revista Bem Legal, v.4, n.2, 2014.

SILVA, C. F.; FERREIRA, S.B.L.; SACRAMENTO, C. Mobile Application Accessibility in the Context of Visually Impaired Users. Association for Computing Machinery New York NY United States; 2018.

SOARES, K. R. B. M-learning. O uso do aplicativo Duolingo para o ensino de gramática e vocabulário em língua inglesa em curso técnico de nível médio. 2018. Dissertação (Mestrado em Ensino) -Programa de Pós-Graduação em Ensino (UERN, UFERSA, IFRN), Instituto Federal do Rio Grande do Norte, Mossoró, 2018.

SOARES, K. R. B.; LIMA, S. de C. O uso do Duolingo no ensino de língua inglesa em curso técnico de nível médio integrado. Revista LínguaTec. v. 3, n. 1, p. 158-170, jun. 2019.

VOLLER, P. (Eds.). Autonomy and Independence in Language Learning. London: Longman, 1997, p. 18-34.

\section{Recebido em: 15/12/2021 \\ Aprovado em: 18/01/2022 \\ Publicado em: 23/01/2022}

\section{AUTORES:}

\section{Poliane Pimentel dos Anjos}

Especialista em Informática Aplicada a Educação pela Universidade do Estado do Amazonas, Licenciada em Lingua Inglesa pela Universidade Paulista -UNIP, Docente na E.E. Balbina Mestrinho E-mail: poliane.pimentel@gmail.com 


\section{Prisna Jamile Santos Leder}

Graduaada em Ciências Naturais - Química pela Universidade do Estado do Pará UEPA.

Especialiata em Ensino de Química pela Faculdade Única.

Mestra em Tecnologia de Processos Químicos e Bioquímicos pela Universidade Tecnológica Federal do Paraná; Docente na Escola Estadual Balbina Mestrinho, SEDUCAM, Urucará

E-mail: prisnajamile@gmail.com

\section{Bruna Kerolly Epifanio Martins}

Estudante do $7^{\circ}$ ano da E.E. Balbina Mestrinho

E-mail: brunamartins.pce2021@gmail.com

\section{Daniela Sias}

Estudante do $7^{\circ}$ ano da E.E. Balbina Mestrinho

E-mail: danielasias.pce2021@gmail.com

\section{Paula Vitória Matos Menezes}

Estudante do $7^{\circ}$ ano da E.E. Balbina Mestrinho

E-mail: paula.pce2021@gmail.com

\section{Rafael Silva dos Santos Júnior}

Estudante do $7^{\circ}$ ano da E.E. Balbina Mestrinho

E-mail: rafaelsantos.pce2021@gmail.com 